\title{
The Anisotropic 3D Ising Model
}

\author{
H.J.W. Zandvliet and A. Saedi \\ Physical Aspects of Nanoelectronics, MESA+ Institute for Nanotechnology, \\ University of Twente, P.O. Box 217, 7500 AE Enschede, The Netherlands \\ C. Hoede \\ Department of Applied Mathematics, University of Twente, P.O. Box 217, 7500 \\ AE Enschede, The Netherlands
}

An asymptotically exact expression for the free energy of an (001) oriented domain wall of the 3D Ising model is derived. The order-disorder transition takes place when the domain wall free energy vanishes. In the anisotropic limit, where two of the three exchange energies (e.g. $J_{X}$ and $J_{y}$ ) are small compared to the third exchange energy $\left(J_{Z}\right)$, the following equation for the critical temperature is derived, $\sinh \left(\frac{2 J_{Z}}{k_{B} T_{C}}\right) \sinh \left(\frac{2\left(J_{X}+J_{y}\right)}{k_{B} T_{C}}\right)=1$. It is shown that this expression is asymptotically exact.

PACS numbers: 68.35.Rh Phase transitions and critical phenomena, 75.40.-s Critical point effects, specific heats, short-range order 


\section{Introduction}

In contrast to the $1 \mathrm{D}$ and the 2D Ising models, the 3D Ising model has not been solved exactly. There is great interest in the 3D Ising model, because it can be used to describe a broad class of phase transitions, e.g. binary alloys, simple liquids and their mixtures, polymer solutions, easy-axis magnets etc. [1,2].

Griffiths provided the first rigorous proof that the Ising model in three dimensions exhibits a phase transition in the sense of having a nonzero spontaneous magnetization below some critical temperature $T_{C}$ [3]. In his approach he made use of a heuristic argument that has been put forward and applied by Peierls to the 2D case [4]. In 1967 Weng, Griffiths and Fisher [5] derived a lower bound on the critical temperature of the anisotropic 3D Ising model that is given by

$$
\frac{k_{B} T_{C}}{J_{z}}=2\left[\ln \left(\frac{J_{z}}{J_{x}+J_{y}}\right)-\ln \left(\ln \left(\frac{J_{z}}{J_{x}+J_{y}}\right)\right)+O(1)\right]^{-1}
$$

This expression predicts a logarithmic decrease in the critical temperature with increasing exchange energy anisotropy. Weng, Griffiths and Fisher [5] surmised that the above equation is even asymptotically exact. The latter was indeed confirmed in a subsequent paper by Fisher [6]. In that paper Fisher derived an upper bound on the critical temperature, which has the exact form of Eq. (1). More recently, Yurishchev [7] derived upper and lower bounds on the critical temperature by using the transfer-matrix technique and an extended phenomenological renormalization group theory approach $[8,9]$. Yurishchev 
showed that for a relatively small anisotropy $\left(<10^{-3}\right)$ the accuracy of Eq. (1) is rather low. However, it should be pointed out that this by no means implies that Eq. (1) is not valid. Weng, Griffiths and Fisher [5] and Fisher [6] clearly pointed out that: (i) Eq. (1) is only asymptotically exact and (ii) the exact value of $\mathrm{O}(1)$ is not known.

It is the aim of this paper to put forward an alternative way to estimate the critical temperature of the 3D Ising lattice. The method relies on finding an approximate expression for the domain wall free energy [10-13]. The domain wall separates two regions of opposite spin ordering. The temperature at which the domain wall free energy vanishes is the critical temperature. The expression we construct for the domain wall free energy is asymptotically exact in the anisotropic limit, where two of the three exchange energies vanish.

\section{Results and Discussion}

We consider the anisotropic simple-cubic Ising lattice. The nearest-neighbor exchange energies between the spins in the $x-, y$ - and $z$ - direction are represented by $J_{X}, J_{y}$ and $J_{Z}$, respectively. For convenience we use the following notation,

$H_{X}=\frac{J_{X}}{k_{B} T}, \quad H_{y}=\frac{J_{y}}{k_{B} T}$ and $H_{Z}=\frac{J_{Z}}{k_{B} T} \quad$,

where $k_{B}$ is Boltzmann's constant and $T$ the temperature. For the sake of simplicity we assume that all the nearest neighbor interactions are ferromagnetic, i.e. $J_{X, y, z}>0$. In order to derive an expression for the order-disorder phase transition temperature we consider 2 domains with opposite spin ordering. A 
similar approach has been applied to the 2D rectangular Ising lattice with nearest- and next-nearest neighbors [10-13]. We assume that at zero temperature the domain wall is located in the $x y$-plane. It should be pointed out here that this choice does not have any influence on our main conclusion. The procedure we will follow here is that we will derive an expression for the partition function per spin of the domain wall. The order-disorder temperature $\left(T_{C}\right)$ can subsequently be found by requiring that $F=-k_{B} T_{C} \ln (Z)=0$ [14].

At zero temperature the domain wall is flat, however with increasing temperature excitations in the domain wall will appear. With increasing temperature the energy required for the generation of these excitations is overcome by an increase in entropy. Each cell $(i, j)$ of the domain wall is represented by a column in the $z$-direction with height $h(i, j)$ and is surrounded by four neighbors with indices $(i-1, j),(i+1, j),(i, j-1)$ and $(i, j+1)$ respectively (see Fig. 1). As is evident the height differences between these columns directly affect the formation energy of the domain wall. The total partition sum of the domain wall, $Z_{\text {tot }}$, can be written as,

$$
Z_{\text {tot }}=\sum_{m} e^{-E_{m} / k_{B} T}=\prod_{(i, j)} Z_{(i, j)}
$$

where the summation $m$ runs over all possible configurations of the domain wall and $Z_{(i, j)}$ is the partition sum of the $(i, j)$-th cell. We start with the assumption that $H_{Z} \gg>H_{X, y}$. If overhangs and inclusions (i.e. droplets and bubbles of the opposite spin ordering) are ignored the following partition function of $(i, j)$-th cell, $Z_{(i, j)}$, can be derived; 


$$
Z_{(i, j)}=\sum_{h(i, j)=-\infty}^{\infty} e^{-\left(2 H_{Z}+2 H_{X}|h(i, j)-h(i-1, j)|+2 H_{y}|h(i, j)-h(i, j-1)|\right)}
$$

The summation in Eq. (4) can be separated into three terms

$$
\begin{aligned}
& Z_{(i, j)}=e^{-2 H_{Z}} \sum_{h(i, j)=-\infty}^{-1} e^{2 h(i, j)\left(H_{X}+H_{y}\right)-2|\bar{h}| H_{y}}+ \\
& e^{-2 H_{Z}} \sum_{h(i, j)=0}^{|\bar{h}|} e^{-2 h(i, j) H_{X}-2(|\bar{h}|-h(i, j)) H_{y}}+e^{-2 H_{Z}} \sum_{h(i, j)=|\bar{h}|+1}^{\infty} e^{-2(h(i, j)-|\bar{h}|)\left(H_{X}+H_{y}\right)-2|\bar{h}| H_{X}}
\end{aligned}
$$

where $\bar{h}=h(i-1, j)-h(i, j-1)$ (see Fig. 1$)$.

It is convenient to replace $h(i, j)$ by $n$ and rearrange Eq. (5) to

$$
Z_{(i, j)}=e^{-2 H_{Z}}\left[\sum_{n=0}^{|\bar{h}|} e^{-2 n H_{X}-2(|\bar{h}|-n) H_{y}}+\left(e^{-2|\bar{h}| H_{X}}+e^{-2|\bar{h}| H_{y}}\right) \sum_{n=1}^{\infty} e^{-2 n\left(H_{X}+H_{y}\right)}\right]
$$

All three terms deal with the energy that is required to form the side planes of the column $h(i, j)$. The probabilities of finding an upward or downward excitation (see Fig. 1) are equal and therefore the mean overall orientation of the domain wall is maintained parallel to the $x y$-plane. Eq. (6) can be rewritten to 


$$
Z_{(i, j)}=e^{-2 H_{Z}}\left[\left(\frac{e^{-2(|\bar{h}|+1) H_{X}}-e^{-2(|\bar{h}|+1) H_{y}}}{e^{-2 H_{X}}-e^{-2 H_{y}}}\right)+\left(\frac{\left(e^{-2|\bar{h}| H_{X}}+e^{-2|\bar{h}| H_{y}}\right) e^{-2\left(H_{X}+H_{y}\right)}}{1-e^{-2\left(H_{X}+H_{y}\right)}}\right)\right]
$$

It is convenient to introduce the following variables;

$u=e^{-2 H_{X}}, v=e^{-2 H_{y}}$ and $w=e^{-2 H_{Z}}$

Using these varaibles for $u, v$ and $w$ Eq. (7) can be written as

$$
Z_{(i, j)}=w\left[\frac{\left(u^{|\bar{h}|+1}-v^{|\bar{h}|+1}\right)}{(u-v)}+\frac{\left(\left(u^{|\bar{h}|}+v^{|\bar{h}|}\right) u v\right)}{(1-u v)}\right]
$$

Ignoring overhangs and inclusions is appropriate only in the case when $H_{Z}>>H_{X, y}$ (these configurations will lead to additional terms in the partition function which are proportional to $e^{-s H_{Z}}$, with $s \geq 6$ ).

The partition function, $Z_{(i, j)}$, exhibits a maximum for $|\bar{h}|=0$, thus leading to an upper bound on $H_{C}\left(H_{C}=J / k_{B} T_{C}\right)$ and a lower bound on the order-disorder phase transition temperature. For this specific case Eq. (7) reduces to 


$$
Z_{(i, j)}=e^{-2 H_{Z}}\left[\frac{1+e^{-2\left(H_{X}+H_{y}\right)}}{1-e^{-2\left(H_{X}+H_{y}\right)}}\right] \quad \text { or } Z_{(i, j)}=w\left[\frac{1+u v}{1-u v}\right]
$$

The order-disorder transition point can be found by setting $Z_{(i, j)}=1$. One finds

$$
\sinh \left(2 H_{z}\right) \sinh \left(2\left(H_{x}+H_{y}\right)\right)=1
$$

By cyclic permutation two similar expressions are found. The difference in these expressions comes from the choice of the wall which has its normal in either the $x, y$ or $z$ direction. In the anisotropic case it is most convenient to define the domain wall in such a way that the normal of the wall is along the strongest coupling direction. As is evident from Eq. (7) this will lead to a maximum suppression of the contribution of overhangs and inclusions to the partition function. In the asymptotic limit the 3D Ising model gradually converts to the $1 \mathrm{D}$ Ising model and the phase transition temperature approaches zero, and thus the expectation value of $\bar{h}$ gradually approaches zero too. However, even for a nonzero value of $\bar{h}$ Eq. (11) is recovered in the asymptotic limit provided that the product $\bar{h} H_{X, y}<<1$. For sufficiently small values of $H_{X, y}$ all factors $e^{-2 \bar{h} H_{X, y}}$ $\left(=u^{\bar{h}}, v^{\bar{h}}\right)$ in Eq. (7) approach 1 and Eq. (10) will be recaptured.

The critical temperature of the anisotropic 2D Ising ferromagnet is known from Onsager's exact solution [15], i.e. $\sinh \left(2 H_{Z}\right) \sinh \left(2 H_{X}\right)=1$, to vanish asymptotically as 
$2 H_{Z}=\left[\ln \left(\frac{H_{Z}}{H_{X}}\right)-\ln \left(\ln \left(\frac{H_{Z}}{H_{X}}\right)\right)+O(1)\right]$,

where the ratio of the exchange energies for bonds parallel to the $\mathrm{x}$ and $\mathrm{z}$ axes, i.e. $\left(\frac{H_{X}}{H_{Z}}\right)$, approaches zero [5,6]. Weng, Griffiths and Fisher [5] and Fisher [6] have shown that for the simple cubic lattice an asymptotically exact expression of the same form as Eq. (12) is found with the only modification that $H_{X}$ is replaced by $H_{x}+H_{y}$. The latter provides strong evidence that the asymptotically exact formula that describes the critical line, between the ferromagnetic and paramagnetic phase in the anisotropic limit, can be written as

$\sinh \left(2 H_{Z}\right) \sinh \left(2\left(H_{X}+H_{y}\right)\right)=1$

But this is precisely the result we found in the domain wall analysis. In addition, Eq. (11) not only provides a lower bound on the critical temperature but it is even asymptotically exact, because it exhibits the same asymptotic behavior as the expression derived by Weng Griffiths, Fisher [5] and Fisher [6]. Finally, the value of $\mathrm{O}(1)$ can de determined by comparing Eqs. (1) and (11). We found a value for $\mathrm{O}(1)$ a value that gradually decreases from $\sim 0.84$ at $\left(\frac{H_{X}+H_{y}}{H_{z}}\right)=10^{-2}$ to $\sim 0.76$ at $\left(\frac{H_{x}+H_{y}}{H_{z}}\right)=10^{-20}$.

In summary, we have derived an expression for the critical temperature of the 3D Ising model, which is asymptotically exact in the limit of vanishing exchange energies in two of the three directions. 


\section{References}

[1]. J. Cardy, Scaling and Renormalization in Statistical Physics. Cambridge Univ. Press, Cambridge, (1997).

[2]. A. Pelissetto and E. Vicari, Phys. Rep. 368, 549 (2002).

[3]. R.B. Griffiths, Phys. Rev. 136, A437 (1964).

[4]. R. Peierls, Proc. Cambridge Phil. Soc. 32, 477 (1936).

[5]. C.-Y. Weng, R.B. Griffiths and M.E. Fisher, Phys. Rev. 162, 475 (1967).

[6]. M.E. Fisher, Phys. Rev. 162, 480 (1967).

[7]. M.A. Yurishchev, Journal of Experimental and Theoretical Physics 98, 1183 (2004).

[8]. M.A. Yurishchev, Nucl. Phys. B (proc. Suppl.) 83/84, 727 (2000).

[9]. M.A. Yurishchev, JETP 91, 332 (2000).

[10]. E. Müller-Hartmann and J. Zittartz, Z. Physik B 27, 261 (1977).

[11]. T.W. Burkhardt, Z. Physik B 31, 183 (1978).

[12]. B.W. Southern, Z. Physik B 30, 61 (1978).

[13]. H.J.W. Zandvliet, Europhys. Lett. 73, 747 (2006).

[14]. H.J.W. Zandvliet, Rev. Mod. Phys. 72, 593 (2000).

[15]. L. Onsager, Phys. Rev. 65, 117 (1944). 


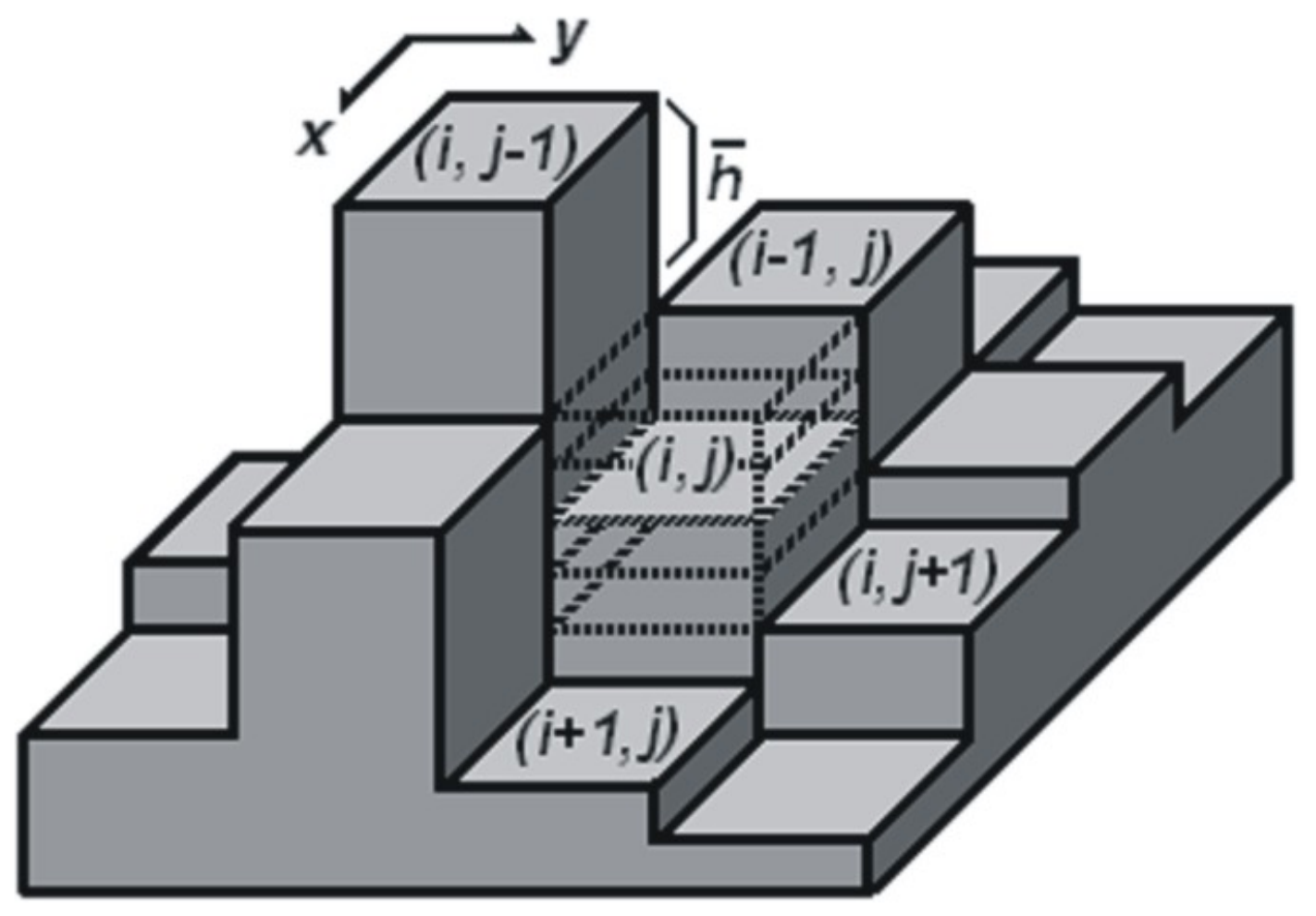

\section{Figure 1}

Schematic model of an $x y$-domain wall. The normal of the domain wall is oriented in the $z$-direction. Each column, $h(i, j)$, is surrounded by four nearest neighbors labeled $h(i-1, j), h(i, j-1), h(i+1, j)$ and $h(i, j+1)$, respectively. $\bar{h}$ is the height difference between the $h(i-1, j)$ and $h(i, j-1)$ columns. $h(i, j) \in[-\infty, \infty](h(i, j)=0$ corresponds to the height of the (i-1,j)-th column). 